\title{
Elastin cross-linking in the skin from patients with amyotrophic lateral sclerosis
}

\author{
Seiitsu Ono, Mitsuo Yamauchi
}

\begin{abstract}
Two cross-links unique to elastin, desmosine and isodesmosine were measured and compared in skin tissue (left upper arm) from 10 patients with amyotrophic lateral sclerosis (ALS) and from seven age-matched controls. The contents of desmosine and isodesmosine were significantly decreased $(p<0.01$ and $p<0.01$, respectively) in patients with ALS compared with those of controls, and were negatively and significantly associated with duration of illness in ALS patients $(r=-0.77, p<0.01$ and $r=-0.65, p<0.05$, respectively). The decline in skin desmosine and isodesmosine is more rapid in ALS than in normal ageing. Thus cross-linking of skin elastin is affected in ALS.
\end{abstract}

(F Neurol Neurosurg Psychiatry 1994;57:94-96)

Several studies have reported an alteration of skin pathology in amyotrophic lateral sclerosis (ALS). ${ }^{1-4}$ Recently it was found that the collagen content of skin from patients with ALS was significantly lower than normal, ${ }^{5}$ and that there was a decrease in the content of nonreducible, stable cross-link, histidinohydroxylysinonorleucine (HHL) and an increase in the content of two reducible iminium cross-links, dehydrohydroxylysinonorleucine (deH-HLNL) and dehydrohistidinohydroxymerodesmosine (deH-HHMD) with duration of illness in the skin collagen of patients with ALS. ${ }^{6}$ However, little is known concerning the biochemical nature of skin elastin in ALS.

Elastic fibres are found in most connective tissue with collagen and polysaccharides, and can stretch to several times their length and then rapidly return to their original size and shape when the tension is released. Elastin is the major component of elastic fibres and therefore ideally suited for this role partly due to a series of lysine-derived, covalent crosslinks. ${ }^{7}$ These cross-links are formed extracellularly and contribute to the remarkable stability of the elastic fibre. Of these crosslinks, desmosine and its isomer, isodesmosine, are unique to elastin in mammalian tissues and thereby, provide a specific marker for this protein. ${ }^{8}$ Although it is known that the initial step in elastin cross-linking involves the conversion of peptidyl lysine to 5-amino5-carboxypentanal by the action of lysyl oxidase ${ }^{9}$ the detailed mechanism of formation of desmosine and isodesmosine is still unknown. Since the same initial step is required in the formation of collagen cross-links which we have found to be disrupted in skin tissue in ALS, ${ }^{6}$ we have now quantified cross-links of elastin in skin at various stages of ALS and compared the results to those of controls.

\section{Patients and methods \\ PATIENTS}

There were 10 patients with ALS (mean age, 59 years; range, 52-66 years) and seven agematched controls with other neurological or muscular diseases (mean age, 61 years; range, 55-71 years). All patients with ALS had the presence of both upper and lower motor neuron signs, clear evidence of progression, normal nerve conduction velocities and late responses, and electromyographic evidence of diffuse denervation. Evaluation included a detailed history and physical examination, and extensive haematological, biochemical, electrophysiological, and radiological testing. At least three neurologists agreed on the diagnosis in each case. The diagnoses in the control group were spinocerebellar degeneration, polymyositis, Alzheimer's disease, Parkinson's disease, cerebral infarction, progressive muscular atrophy, and myasthenia gravis in one patient each. Informed consent was obtained from all patients with ALS and controls. Punch biopsy specimens of skin overlying the left biceps were taken following local anaesthesia with $1 \%$ procaine hydrochloride, and were stored at $-80^{\circ} \mathrm{C}$ until use.

\section{PREPARATION OF SAMPLES}

The skin was carefully trimmed of hair and subcutaneous fat. All preparations were carried out at $4^{\circ} \mathrm{C}$. Samples were pulverised under liquid nitrogen using a Spex Freezer Mill (Spex, Metuchen, New Jersey) to a fine powder, and then washed with cold $0.015 \mathrm{M}$ $\mathrm{N}$-trishydroxymethyl-2-aminoethanesulfonic acid (TES) buffer, $\mathrm{pH} 7 \cdot 4$ to remove some of the soluble material and serum proteins. The insoluble fraction was extensively dialysed against cold distilled water, and lyophilised. 

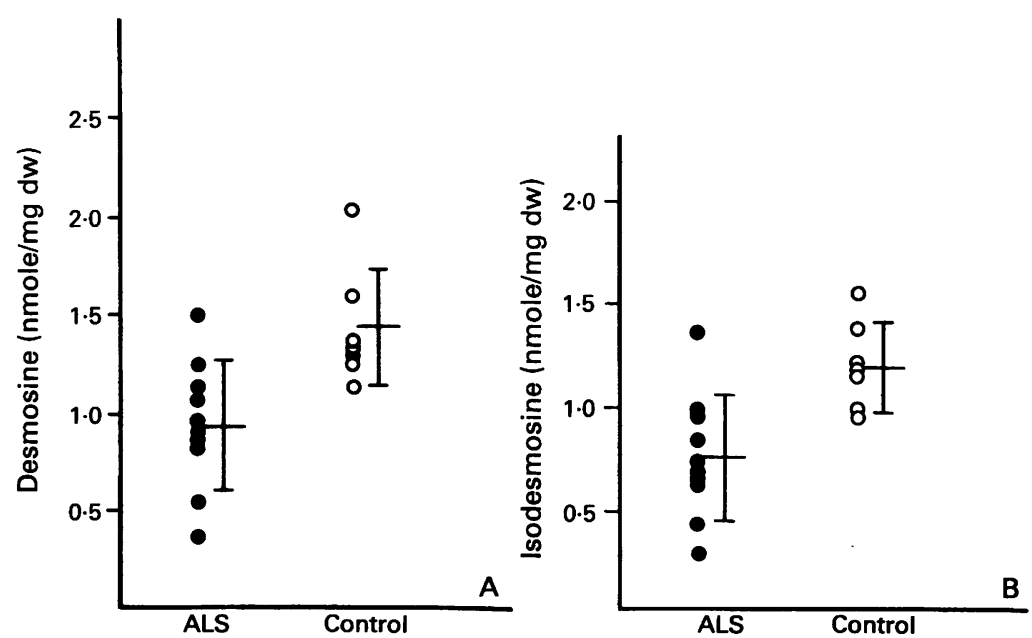

Figure 1 The contents of desmosine $(A)$ and isodesmosine (B) in patients with $A L S$ and control subjects. The contents of desmosine and isodesmosine are significantly decreased in patients with ALS compared with those of control subjects $(p<0.01$ and $p<0.01$, respectively). Bars show mean (SD); dw = dry weight of the sample.

\section{CROSS-LINK ANALYSIS}

Cross-links of desmosine and isodesmosine were analysed by quantitative amino acid analysis. The carefully weighed dried skin powder was hydrolysed with $6 \mathrm{~N} \mathrm{HCl}$ in vacuo in an $\mathrm{N}_{2}$ atmosphere for 24 hours at $110^{\circ} \mathrm{C}$. The hydrolysates were evaporated by speed centrifugation (Savant Instruments) and the residues were dissolved in $0.5-1.0 \mathrm{ml}$ of distilled water. After the sample solution was filtered, desmosine and isodesmosine contents of each sample were directly quantified by an amino acid analyser (Varian 5500 liquid chromatography, AA911 column, Interaction). The ninhydrin reactant was monitored by absorbance at $570 \mathrm{~nm}$ for desmosine and isodesmosine. The ninhydrin colour factors for desmosine and isodesmosine were determined based on the standards which were commercially obtained (Elastin Products Company). The values of desmosine and isodesmosine were expressed as nmoles per mg dry weight of insoluble skin tissue.

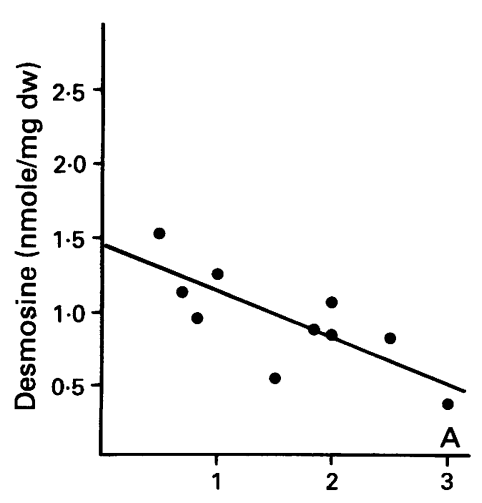

Duration of disease (years)

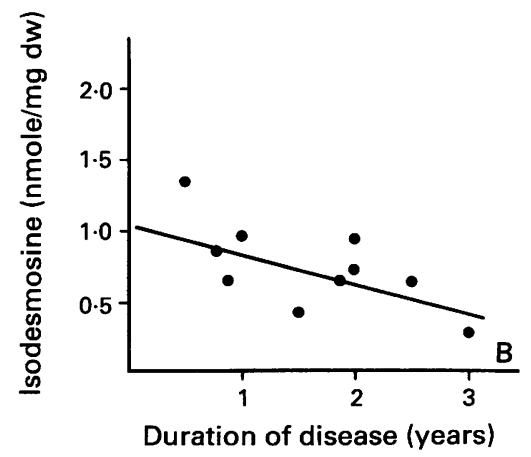

Figure 2 Correlation of duration of illness to the contents of desmosine (A) and isodesmosine $(B)$ in patients with $A L S$. The contents of desmosine and isodesmosine are plotted as a function of duration of illness. There is a significant negative relationship between the contents of desmosine and isodesmosine, and duration of illness $(r=-0.77$, $p<0.01$ and $r=-0.65, p<0.05$, respectively); dw = dry weight of the sample.
Statistical comparisons were made by unpaired Student's $t$ test, with $\mathrm{p}<0.05$ as the significance level. Correlation coefficients were calculated by the least-squares methods.

\section{Results}

The contents of desmosine and isodesmosine were significantly decreased $(p<0.01$ and $\mathrm{p}<0.01$, respectively) in patients with ALS [mean (SD) $0.94(0.33)$ and $0.74(0.30)$ nmoles/mg dry weight; range, $0.36-1.51$ and 0.29-1.33 nmoles/mg dry weight, respectively] compared with those of controls [mean (SD) $1.43(0.30)$ and $1.19(0.21) \mathrm{nmoles} / \mathrm{mg}$ dry weight; range, 1.11-2.03 and 0.92-1.52 nmoles/mg dry weight, respectively] (fig 1). The contents of desmosine and isodesmosine were negatively and significantly associated with the duration of illness in patients with ALS $(r=-0.77, p<0.01$ and $r=-0.65$, $p<0.05$, respectively) (fig 2 ). These data indicated that the amount of mature crosslinks of elastin decreased as a function of duration of disease in ALS. The ratio of desmosine and isodesmosine was relatively constant [mean (SD) $1.29(0.31)$; range, $1 \cdot 18-1 \cdot 41]$ and there was no significant change in the proportion of these two crosslinks in all samples analysed. These ratios were in good agreement with previous reports. ${ }^{10}$

\section{Discussion}

It was reported that the amount of desmosine and isodesmosine decreased in elastins isolated from both aortas and pulmonary tissues of humans with increasing age. ${ }^{11}$ In our study it was demonstrated that the content of desmosine and isodesmosine in ALS patients decreased with duration of illness. The decrease in mature cross-links of skin elastin in ALS patients during illness takes place more rapidly than would be expected in normal ageing.

Fullmer et $a l^{1}$ found degenerative elastic tissues and fragmentation of elastic fibres with altered collagen bundles in the skin of ALS patients. Störtebecker et $a l^{12}$ who studied biopsied samples of the temporal artery in 12 ALS patients and nine age-matched controls, showed that discontinuities, splitting, and multiple fragments of the internal elastic membrane were observed more frequently in ALS patients than in controls. These changes are considered to reflect normal ageing processes, ${ }^{13}$ suggesting that morphological changes caused by the ageing process in the elastic component occur more quickly in ALS patients than in controls. The biochemical data presented in this report are consistent with these morphological findings in ALS patients.

The content of desmosine in various elastin preparations has been shown to be fairly constant. ${ }^{14}$ Consequently, assay of desmosine and isodesmosine, which account for about 1.5 and 1.0 residues per 1000 
amino acids, respectively, can be used as a measure of the amount of elastin in tissues. ${ }^{14}$ Based on this assumption, it is likely that there is a marked decrease in the content of elastin in the skin of patients with ALS. Because the immediate precursors of desmosine and isodesmosine are not known, it is not possible at present to ascertain whether these precursors increase as desmosine and isodesmosine decrease, in a manner similar to HHL cross-linking in collagen of ALS skin. ${ }^{6}$

The presumed mechanism of elasticity of tissues is based on the continuity of the network of elastin polypeptides interconnected by stable cross-linkages predominantly in the form of desmosine and isodesmosine. Alterations in the supramolecular organisation of the elastic fibre network could lead to mechanical alterations in the skin, which would manifest as wrinkled, loose, and sagging skin, as noted during cutaneous ageing. ${ }^{15}$ In support of this concept are several observations made on patients with either inherited or acquired cutaneous diseases with elastic fibre abnormalities. Cutis laxa is characterised by decreased elasticity of skin due to a decrease in skin elastin detected by reduced desmosine content, and stretching of the skin of this disease produces delayed recoil. ${ }^{16}$ The skin of ALS patients in its late stage loses elasticity. ${ }^{2-4}$ When the skin is stretched, it returns very slowly to its original position ("delayed return phenomenon"), ${ }^{24}$ similar to that found in cutis laxa. ${ }^{15}$ Accordingly, a marked decrease in the amount of elastic fibres in the skin of ALS could well explain this specific clinical finding of ALS patients.

This work was partly supported by NIH grants DE 08522 , DE 08611, AR 19969, AR 30587, and NASA grant NAG-2181.
We thank Dr David L McIlwain of the Department of Physiology of the University of North Carolina at Chapel Hill for his valuable comments and encouragement, and $D$ Theodore A Walter and Mr Z Haque for their technical assis-
tance.

1 Fullmer HM, Siedler HD, Krooth RS, Kurland LT. A cutaneous disorder of connective tissue in amyotrophic cutaneous disorder of connective tissue in amyotrophic 10:717-24.

2 Ono S, Toyokura Y, Mannen $\mathrm{T}$, Ishibashi $\mathrm{Y}$. Amyotrophic lateral sclerosis: histologic, histochemical, and ultrastructural abnormalities of skin. Neurology 1986;36:948-56

3 Ono S, Toyokura Y, Mannan T, Ishibashi Y. "Delayed return phenomenon" in amyotrophic lateral sclerosis. Acta Neurol Scand 1988;77:102-7.

4 Ono S, Mannen T, Toyokura Y. Differential diagnosi between amyotrophic lateral sclerosis and spinal muscular atrophy by skin involvement. F Neurol Sci 1989;91: 301-10.

5 Ono S, Mechanic GL, Yamauchi M. Amyotrophic lateral sclerosis: unusually low content of collagen in skin. f Neurol Sci 1990;100:234-7.

6 Ono S, Yamauchi M. Collagen cross-linking of skin in patients with amyotrophic lateral sclerosis. Annu Neurol patients with amy

7 Piez KA. Cross-linking of collagen and elastin. Annu Rev Biochem 1968;37:547-70.

8 Starcher BC, Percival S. Elastin turnover in the rat uterus. Connect Tissue Res 1985;13:207-15.

9 Francis G, John R, Thomas J. Biosynthetic pathway of desmosines in elastin. Biochem $\mathcal{f} 1973 ; 136: 45-55$.

10 Gunja-Smith Z, Lin J, Woessner JF. Changes in desmo sine and pyridinoline crosslinks during rapid synthesis and degradation of elastin and collagen in the rat uterus. Matrix 1989;9:21-7.

11 John R, Thomas J. Chemical compositions of elastins isolated from aortas and pulmonary tissues of humans of different ages. Biochem $\mathcal{f}$ 1972;127:261-9.

12 Störtebecker $P$, Nordström G, Pestény MP, Seeman T, Björkerud S. Vascular and metabolic studies of amyotrophic lateral sclerosis: angiopathy in biopsy speciotrophic lateral sclerosis: angiopathy in biopsy speci-

13 mens of peripheral arteries. Neurology 1970;20:1157-60. WAD, Kissane JM, eds. Pathology, vol 1, 7th edn. Saint WAD, Kissane JM, eds. Patholo

14 Starcher BC. Determination of the elastin content of tissues by measuring desmosine and isodesmosine. Anal sues by measuring desm
Biochem 1977;79:11-5.

15 Uitto J. Connective tissue biochemistry of the aging dermis: age-related alterations in collagen and elastin. Dermatol Clin 1986;4:433-46.

16 Hashimoto K, Kanzaki T. Cutis laxa: ultrastructural and biochemical studies. Arch Dermatol 1975;111:861-73. 